\title{
Development of oral health-related self-efficacy scale for the cancer patients
}

\section{Yuhei Matsuda ${ }^{1}$, Masaaki Karino', Takahiro Kanno ${ }^{1}$}

${ }^{1}$ Department of Oral and Maxillofacial Surgery, School of Medicine, Shimane University, Shimane, Japan

\section{Introduction}

Self-efficacy is an individual's expectation that determines how well he or she can implement the actions necessary for producing certain results.

It is reported that the oral health-related self-efficacy scale for the patients with periodontitis and geriatrics was developed.

\section{There is no self-efficacy scale for the cancer patients.}

\section{Purpose of this study}

This study developed the Oral health-related Self-Efficacy scale for the Cancer patients (OSEC), which contains 17 questions with four response options each.

\section{Methods}

Between July 2018 and May 2019, 203 participants were enrolled in the study. We conducted the survey to examine the validity and reliability of OSEC. Concurrent validity was determined by correlations with the self-efficacy scale for advanced cancer (SEAC). Discriminant validity was examined using good-poor analysis. Predictive validity was examined using plaque control record.

This study was conducted with the approval of the Medical Ethics Committee of Shimane University Faculty of Medicine (No.3243). We calculated two-tailed p-values in all of the analyses. The alpha level of significance was set at 0.05 .

\begin{tabular}{lll} 
ReSUate & \\
Demographic and clinical characteristics of patients & $(\mathbf{N}=\mathbf{2 0 3})$ \\
\hline Age & Male & $69.6(11.3)$ \\
Sex & Female & $129(63.5)$ \\
Primary tumor site & Stomach & $74(36.5)$ \\
& Colorectal & $28(13.8)$ \\
& Liver & $25(12.3)$ \\
& Lung & $11(5.4)$ \\
& Prostate & $25(32.0)$ \\
& Breast & $17(8.4)$ \\
Stage & Head and Neck & $36(17.7)$ \\
& I & $78(38.4)$ \\
& II & $39(19.2)$ \\
& III & $42(20.7)$ \\
IV & $44(21.7)$ \\
\hline
\end{tabular}

Promax rotated factor loadings for construct validity and internal consistency $(\mathrm{N}=\mathbf{2 0 3})$

Factor 1 . Oral function efficacy (OFE)
I can talk smoothly.
I can swallow easily even without a drink or soup.
I can chew anything without a problem.
I can talk with others without worrying about my mouth.
Factor 2. Dental visit efficacy (DVE)
I can go for routine checkups for oral health.
I can continue visiting the clinic periodically even after
treatment to prevent a recurrence.
I can go for routine checkups even after discharged
hospital.
Factor 3 . Adverse effect efficacy (AEE)
I can use special techniques to relieve the symptoms when
the condition of my mouth change.
I can check the change and abnormality of my mouth.
I can keep clean my mouth when the condition of mouth
change.
I can keep clean my mouth even when I feel heavy.
Factor 4 . Symptom coping efficacy (SCE)
I can listen to and follow necessary advice for oral adverse
effect.
I can use a medicine and oral care tools to relieve the oral
adverse effect.
I can consult medical staff on how to relieve the oral
adverse effect.
Factor 5 . Brushing habit efficacy (BHE)
I can use special techniques to brush my teeth.
I can brush my teeth thoroughly.
I can check the cleanliness of my mouth.
Sum of squares on factor loading
Variance explained (\%)
Cumulative variance explained (\%)
Cronbach's a

\begin{tabular}{|c|c|c|c|c|}
\hline \multicolumn{5}{|c|}{ Factor loaaling } \\
\hline 1 & 2 & 3 & 4 & 5 \\
\hline 0.84 & 0.10 & 0.39 & 0.17 & 0.20 \\
\hline 0.79 & 0.05 & 0.17 & 0.30 & 0.15 \\
\hline 0.76 & 0.06 & 0.22 & 0.12 & 0.19 \\
\hline 0.75 & 0.19 & 0.38 & 0.21 & 0.33 \\
\hline 0.08 & 0.93 & 0.35 & 0.53 & 0.26 \\
\hline 0.16 & 0.91 & 0.35 & 0.54 & 0.23 \\
\hline 0.09 & 0.84 & 0.36 & 0.41 & 0.36 \\
\hline 0.34 & 0.24 & 0.85 & 0.44 & 0.24 \\
\hline 0.30 & 0.30 & 0.79 & 0.36 & 0.36 \\
\hline 0.23 & 0.44 & 0.76 & 0.41 & 0.54 \\
\hline 0.20 & 0.34 & 0.74 & 0.44 & 0.47 \\
\hline 0.12 & 0.51 & 0.39 & 0.89 & 0.28 \\
\hline 0.30 & 0.44 & 0.51 & 0.85 & 0.30 \\
\hline 0.25 & 0.52 & 0.52 & 0.85 & 0.34 \\
\hline 0.28 & 0.26 & 0.45 & 0.29 & 0.86 \\
\hline 0.29 & 0.18 & 0.43 & 0.30 & 0.85 \\
\hline 0.10 & 0.32 & 0.27 & 0.19 & 0.73 \\
\hline 6.00 & 2.30 & 1.55 & 1.21 & 0.90 \\
\hline 35.1 & 13.5 & 9.10 & 7.10 & 5.23 \\
\hline 35.1 & 48.7 & 57.8 & 64.9 & 70.1 \\
\hline 0.75 & 0.79 & 0.88 & 0.84 & 0.80 \\
\hline
\end{tabular}

\section{Conclusion}

We developed the OSEC as a multidimensional oral health-related self-efficacy scale for the cancer patients.
Relationship between OSEC and self-efficacy scale for advanced cancer (SEAC) for concurrent validity.

\begin{tabular}{|c|c|c|c|c|c|c|c|}
\hline & OSEC & OFE & DVE & AEE & SCE & BHE & Total \\
\hline \multicolumn{8}{|l|}{ SEAC } \\
\hline \multirow{2}{*}{ ARE } & $r$ & $0.37^{* *}$ & $0.40^{* *}$ & $0.41^{* *}$ & $0.46^{* *}$ & $0.25^{* *}$ & $0.54^{* *}$ \\
\hline & P-value & $<0.01$ & $<0.01$ & $<0.01$ & $<0.01$ & $<0.01$ & $<0.01$ \\
\hline \multirow{2}{*}{ SCE } & $r$ & $0.39^{* *}$ & $0.41^{* *}$ & $0.48^{* *}$ & $0.49^{* *}$ & $0.27^{* *}$ & $0.58^{* *}$ \\
\hline & $\mathrm{P}$-value & $<0.01$ & $<0.01$ & $<0.01$ & $<0.01$ & $<0.01$ & $<0.01$ \\
\hline \multirow{2}{*}{$A D E$} & $r$ & $0.43^{* *}$ & $0.33^{* *}$ & $0.39^{* *}$ & $0.45^{* *}$ & $0.19^{* *}$ & $0.51^{* *}$ \\
\hline & $\mathrm{P}$-value & $<0.01$ & $<0.01$ & $<0.01$ & $<0.01$ & $<0.01$ & $<0.01$ \\
\hline \multirow{2}{*}{ SE (Total) } & $r$ & $0.42^{* *}$ & $0.40^{* *}$ & $0.45^{* *}$ & $0.49^{* *}$ & $0.25^{* *}$ & $0.57^{* *}$ \\
\hline & $\mathrm{P}$-value & $<0.01$ & $<0.01$ & $<0.01$ & $<0.01$ & $<0.01$ & $<0.01$ \\
\hline
\end{tabular}

Test-retest reliability of oral health-related self-efficacy scale for the cancer patients by intra-class correlation coefficient. $(\mathbf{N}=\mathbf{2 0})$

\begin{tabular}{cc} 
Factor & ICC $(95 \%$ CI $)$ \\
\hline OFE & $0.82(0.55-0.93)$ \\
DVE & $0.76(0.40-0.91)$ \\
AEE & $0.90(0.74-0.96)$ \\
SCE & $0.86(0.64-0.94)$ \\
BHE & $0.91(0.77-0.96)$ \\
Total & $0.92(0.79-0.97)$ \\
\hline
\end{tabular}

Good-Poor analysis of oral health-related self-efficacy scale for the cancer patients for discriminant validity

\begin{tabular}{|c|c|c|c|c|c|}
\hline \multicolumn{2}{|c|}{ Variable } & Mean (SD) & $95 \%$ CI of difference & $\begin{array}{l}\text { Effect } \\
\text { size }\end{array}$ & $P$ \\
\hline OFE & $\begin{array}{l}\text { High group } \\
\text { Low group }\end{array}$ & $\begin{array}{l}12.0(1.94) \\
9.57(1.91)\end{array}$ & $-2.97--1.91$ & 0.54 & $<0.01$ \\
\hline DVE & $\begin{array}{l}\text { High group } \\
\text { Low group }\end{array}$ & $\begin{array}{l}9.29(1.71) \\
7.22(1.59)\end{array}$ & $-2.52--1.61$ & 0.53 & $<0.01$ \\
\hline AEE & $\begin{array}{l}\text { High group } \\
\text { Low group }\end{array}$ & $\begin{array}{c}11.70(1.66) \\
9.16(1.53)\end{array}$ & $-2.99--2.10$ & 0.62 & $<0.01$ \\
\hline SCE & $\begin{array}{l}\text { High group } \\
\text { Low group }\end{array}$ & $\begin{array}{l}9.34(1.22) \\
7.80(1.39)\end{array}$ & $-1.90--1.18$ & 0.51 & $<0.01$ \\
\hline BHE & $\begin{array}{l}\text { High group } \\
\text { Low group }\end{array}$ & $\begin{array}{l}8.64(1.32) \\
6.72(1.53)\end{array}$ & $-2.32--1.51$ & 0.55 & $<0.01$ \\
\hline Total & $\begin{array}{l}\text { High group } \\
\text { Low group }\end{array}$ & $\begin{array}{l}50.98(4.74) \\
40.48(3.55)\end{array}$ & $-11.64--9.35$ & 0.79 & $<0.01$ \\
\hline
\end{tabular}

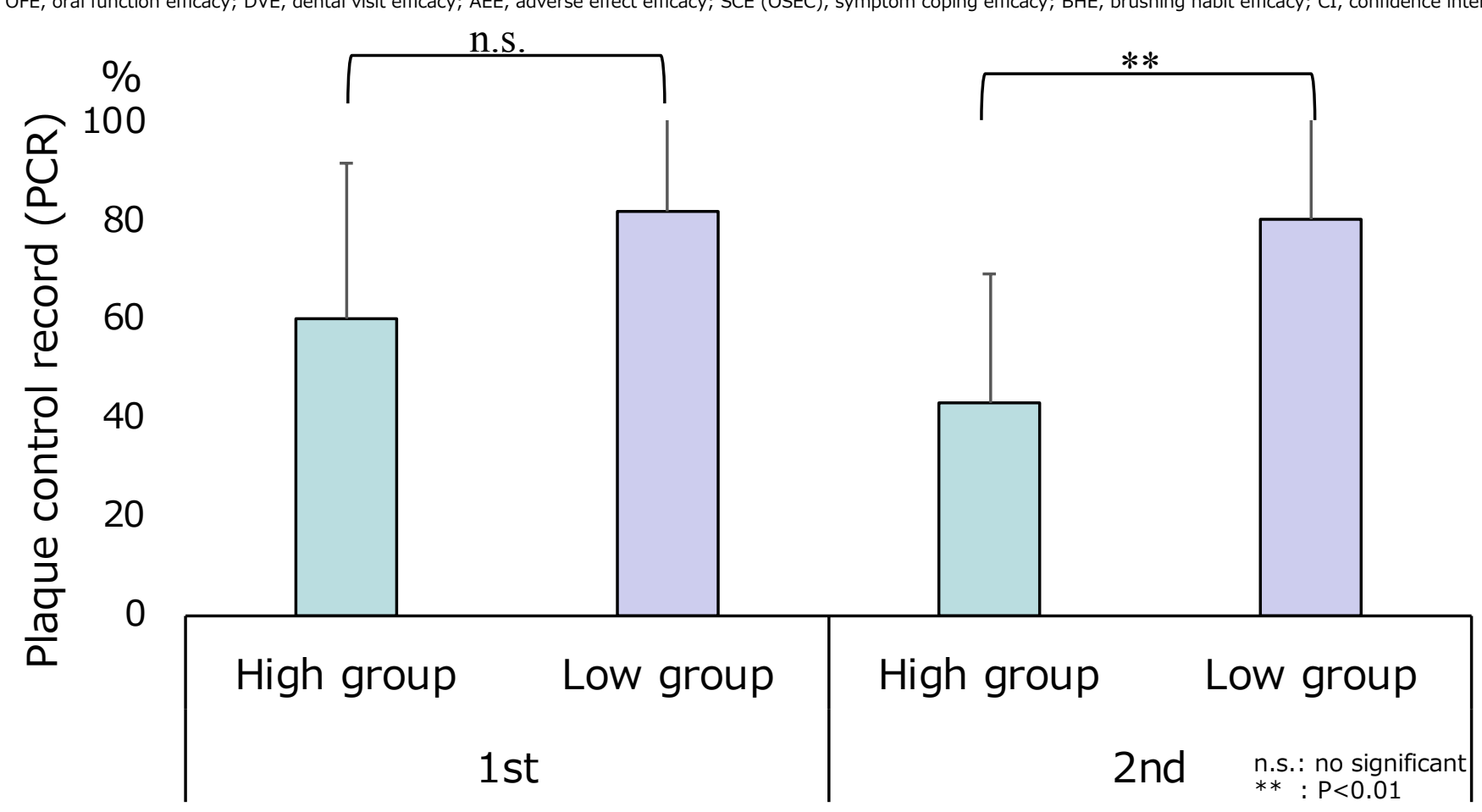

Predictive validity at the $1^{\text {st }}$ and $2^{\text {nd }}$ time comparing between high and low group of plaque control record by t-test $(\mathrm{N}=26)$

\section{Discussion}

- OSEC shows a multidimensional scale (five dimensions).

- Cronbach's a and ICC as reliability comparable to other self-efficacy scale.

The relationship between OSEC and SEAC resulted in reasonable values.

Good-poor analysis showed that there were significant differences between all subscale.

OSEC have a predictive capability for brushing habits.

The validity and reliability of OSEC was certified. Future study

A exploratory study to search related factors of OSEC is needed.

\section{Reference}

1. Geriatr Gerontol Int. 17(10): 1406-1411, 2017

2. J Eval Clin Pract. 17(6): 1023-1029

3. Peiatr Dent. 32(4): 310-315, 2010

4. Psychooncology. 11(3): 221-229, 2002 